\title{
Moving Object Detection Between Multiple and Color Images
}

\author{
Emrullah Durucan \\ DaimlerChrysler AG \\ RIC/AP, HPC T728 \\ 70546 Stuttgart, Germany \\ Email: emrullah.durucan@daimlerchrysler.com
}

\author{
Touradj Ebrahimi \\ Swiss Federal Institute of Technology \\ Signal Processing Laboratory \\ 1015 Lausanne, Switzerland \\ Email: touradj.ebrahimi@epfl.ch
}

\begin{abstract}
There are several publications dedicated to the description and analysis of change detection between two gray-value images. This paper now introduces new methods to detect moving objects between multiple images and to detect changes between color images or any type of multispectral images. We are not aware of methods giving the possibility to detect color changes and changes between multiple frames.
\end{abstract}

All proposed change detectors in this chapter are based on the Gramian determinant, which provides low computational cost and is easy to implement. These features are very important due to the additional complexity of change detection between multiple as well as color images.

\section{INTRODUCTION}

In this paper, we want to concentrate on the detection of moving objects between multiple images and to the detection of changes between two color images. This is in fact the novelty in this paper since it introduces a concise and smart but generalized method to detect changes in any kind of images and not only between two gray-value. In fact, we are not aware of comparable state-of-the-art methods which enable the detection of changes between several frames and color images.

This paper presents in fact a possible extension of the previously presented concept of linear independence [5], [2] which was based on the detection of changes in gray-valued images. These papers also present comparisons to other state-of-theart change detection methods, detecting changes between two gray-valued images [10], [8], [14], [16], [1], [6], [11] .

We do not claim to provide an exhaustive investigation on color spaces and color change detection which is in fact beyond the scope of this paper. An exhaustive study on color spaces and color imaging can be found in [13], [17].

In this paper we will not present the original images used to present the performance of the proposed methods. However these images including an exhaustive description of their contents could be found in [2]. Finally, the definition of adaptive thresholds can be found in [4], [3].

In previous papers we have proposed to represent images as an ensemble of vectors [4], [3]. By modelling the image as an ensemble of vectors, changes in their lengths or directions (with respect to the initial unchanged status) can be exploited. We assume that a change of illumination between two images results in a change of a vector's length (scaling) and other changes result in a change in the vector's direction. Mathematically, this corresponds to a change in the degree of linear dependence between the original and the changed vector. A suitable linear dependence measure can be used as the basis for change detection. In fact the Gramian Matrix provides a necessary and sufficient condition for the linear independence of vectors. The theoretical basis is presented in Section II.

The new multi image moving object detector will be presented in Section III.

In Section IV we will motivate the choice of the color space. In Sections IV-A we will introduces the Color Pixel Gramian for color pixel change detection, where all pixels are considered as vectors of the RGB color space[7], [15]:

$$
x=\left(\begin{array}{l}
r_{x} \\
g_{x} \\
b_{x}
\end{array}\right) ; y=\left(\begin{array}{l}
r_{y} \\
g_{y} \\
b_{y}
\end{array}\right)
$$

where $x, y$ originates from a reference and a current image respectively.

Finally, we will propose the Vector Color Pixel Gramian method IV-B which considers all $x$ and $y$ color vectors as components of the previously presented vectors $u=\left(x_{i}, \cdots, x_{n}\right)$ and $v=\left(y_{i}, \cdots, y_{n}\right)$. The theory and implementation issues, along with the change detection results, are provided for the proposed change detectors.

\section{LineAR DEPENDENCE AND THE GRAMIAN MATRIX}

In this section we would like present to present the theoretical motivation for the use of the Gramian Matrix.

Theorem 1: Let $\mathcal{V}$ be a vector space over a field $\mathbb{K}$ and $():, \mathcal{V} \times \mathcal{V} \rightarrow \mathbb{K}$ a definite scalar product on $\mathcal{V}$. For a finite sequence $\left\{u_{i}\right\}_{i=1}^{m}$ of vectors, let $G=\left(\left(u_{i}, u_{j}\right)\right) \in \mathbb{K}^{m \times m}$ be 
the Gramian matrix of $\left\{u_{i}\right\}_{i=1}^{m}$ :

$$
G=\left(\begin{array}{llll}
\left(u_{1}, u_{1}\right) & \left(u_{1}, u_{2}\right) & \ldots & \left(u_{1}, u_{m}\right) \\
\left(u_{2}, u_{1}\right) & \left(u_{2}, u_{2}\right) & \ldots & \left(u_{2}, u_{m}\right) \\
\cdots & \ldots & \ldots & \ldots \\
\left(u_{m}, u_{1}\right) & \left(u_{m}, u_{2}\right) & \ldots & \left(u_{m}, u_{m}\right)
\end{array}\right)
$$

Then the following three conditions

(1) $\left\{u_{i}\right\}_{i=1}^{m}$ are linearly independent,

(2) $\operatorname{det} G>0$,

(3) $\operatorname{det} G \neq 0$,

are equivalent. For a proof of this theorem refer to Huppert [9].

For the mentioned vectors $u$ and $v$, the definition of a change detector is straightforward.

$$
\begin{aligned}
\operatorname{det} G=\left|\begin{array}{ll}
(u, u) & (u, v) \\
(v, u) & (v, v)
\end{array}\right| & =(u, u)(v, v)-(u, v)^{2} \\
& =\sum_{i=1}^{n} x_{i}^{2} y_{i}^{2}-\left|\sum_{i=1}^{n} x_{i} y_{i}\right|^{2}
\end{aligned}
$$

Where $\operatorname{det} G=0$ if and only if

- $u=v$ that is, no changes occurred between reference and current vectors or

- $u=k v$ that is, an illumination change occurred between the reference and current vectors. We assume hereby that $k$ is a constant illuminance scaling factor.

Theoretically real object changes occur when $\operatorname{det} G>0$. In case $\operatorname{det} G=0$ the vectors from the reference and current image are linearly dependent and hence no object change occurred.

\section{Multi-Frame Gramian (MFG)}

\section{A. Theory of the $M F G$}

In this paragraph the Gramian method is applied to formulate a change detector for multiple images.

The Gramian determinant is not restricted to two vectors and the definition for more than two vectors is straightforward. In this paper however, we restrict ourselves to three vectors, as increasing the number of vectors increases the computational cost of the Gramian and causes longer delays, which is incompatible with real-time video-surveillance.

Let us assume that there are three vectors $u=$ $\left(x_{1}, \cdots, x_{n}\right), \quad v=\left(y_{1}, \cdots, y_{n}\right)$ and $w=\left(z_{1}, \cdots, z_{n}\right)$ originating from a reference and two consecutive current images respectively. The Gramian for the corresponding unit vectors is

$$
\begin{aligned}
|G|= & \left|\begin{array}{lll}
\left(u_{e}, u_{e}\right) & \left(u_{e}, v_{e}\right) & \left(u_{e}, w_{e}\right) \\
\left(v_{e}, u_{e}\right) & \left(v_{e}, v_{e}\right) & \left(v_{e}, w_{e}\right) \\
\left(w_{e}, u_{e}\right) & \left(w_{e}, v_{e}\right) & \left(w_{e}, w_{e}\right)
\end{array}\right| \\
= & \left(u_{e}, u_{e}\right)\left(v_{e}, v_{e}\right)\left(w_{e}, w_{e}\right)+\left(u_{e}, v_{e}\right)\left(v_{e}, w_{e}\right)\left(w_{e}, u_{e}\right) \\
& +\left(u_{e}, w_{e}\right)\left(v_{e}, u_{e}\right)\left(w_{e}, v_{e}\right)-\left(w_{e}, u_{e}\right)\left(v_{e}, v_{e}\right)\left(u_{e}, w_{e}\right) \\
& -\left(w_{e}, v_{e}\right)\left(v_{e}, w_{e}\right)\left(u_{e}, u_{e}\right)-\left(w_{e}, w_{e}\right)\left(v_{e}, u_{e}\right)\left(u_{e}, v_{e}\right) \\
= & 1+2\left(u_{e}, v_{e}\right)\left(v_{e}, w_{e}\right)\left(w_{e}, u_{e}\right) \\
& -\left(w_{e} \cdot u_{e}\right)^{2}-\left(w_{e} \cdot v_{e}\right)^{2}-\left(v_{e} \cdot u_{e}\right)^{2} \\
= & 1+2 \frac{1}{u^{2}} \frac{1}{v^{2}} \frac{1}{w^{2}}(u, v)(v, w)(w, u) \\
& -\frac{1}{w^{2}} \frac{1}{u^{2}}(w, u)^{2}-\frac{1}{w^{2}} \frac{1}{v^{2}}(w, v)^{2}-\frac{1}{v^{2}} \frac{1}{u^{2}}(v, u)^{2} .
\end{aligned}
$$

This equation represents the proposed Multiple Frame Gramian (MFG). Without any estimation of the number of arithmetical operations one can already see that the computational cost is very high for only three vectors. The number of memory accesses, which is higher than for the other methods, also increases computational cost.

The Gramian $|G|$ vanishes when at least two vectors are linearly dependent i.e. objects which enter into a scene and which do not move afterwards will not be detected. Thus this model provides a change detector for dynamic changes. This could be useful for traffic flow analysis, i.e. detection and counting of moving cars. Furthermore, it could be used to analyze objects with mostly uniform illuminance values and which enter into scenes having uniform illuminance values as well. In this case, the reference $u$ and the current vectors $v \& w$ are the scaled versions of one another and so their MFG vanishes as well. Finally, the MFG vanishes in the interior of large or slow moving objects. In this case the two consecutive current vectors $v$ and $w$ cover almost the same part of the object and thus the vectors are linearly dependent, so their Gramian vanishes.

Figure 2 illustrates the flowchart for MFG calculation for three vectors i.e. one reference vector and two successive current vectors.

\section{B. Implementation of the $M F G$}

The Multi-Frame Gramian is calculated in one accumulation summation on the reference and the two current vectors, as can be seen in Fig. 2.

The MFG also requires two parameters i.e. the threshold and the vector dimension as already mentioned. The vector dimensions should be kept as low as possible, otherwise it would result in increasing computational costs. In case the resulting Gramian value is greater than the threshold $T$, a change is detected and the center pixel of the corresponding window is marked as changed in the final mask, otherwise it is assumed to be 0 . The thresholds and vector dimensions applied for the image sequences are given in Table I. 


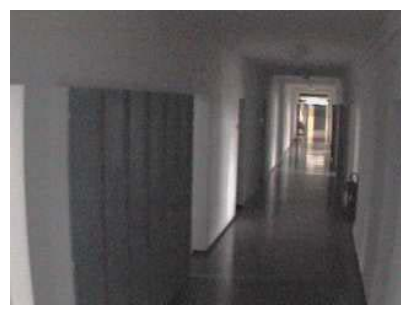

(a) Flur 0

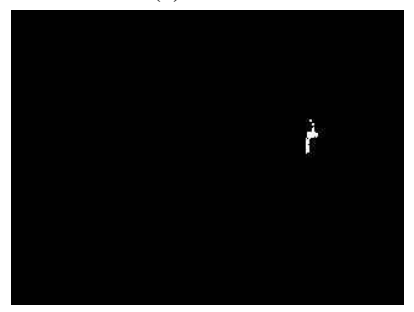

(d) Flur 221

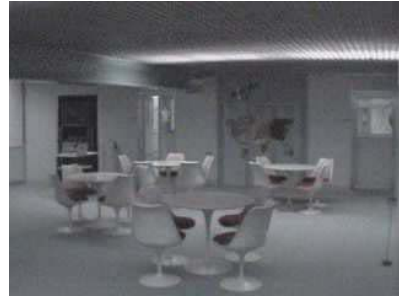

(g) Demoraum 0

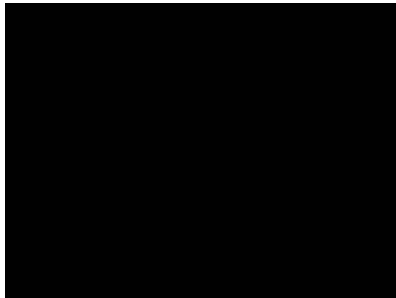

(j) Demoraum 160

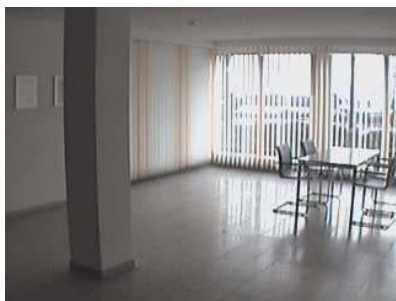

(m) Saeule 0

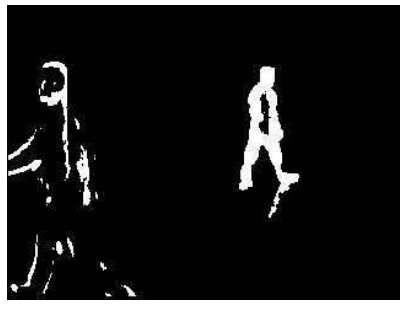

(p) Saeule 310

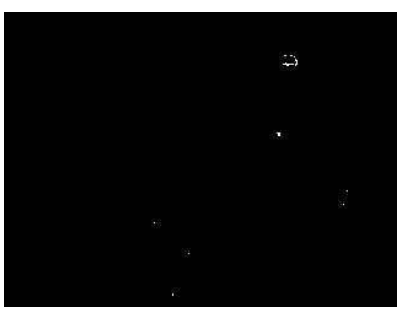

(b) Flur 185

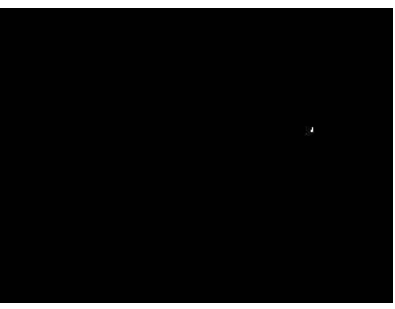

(e) Flur 260

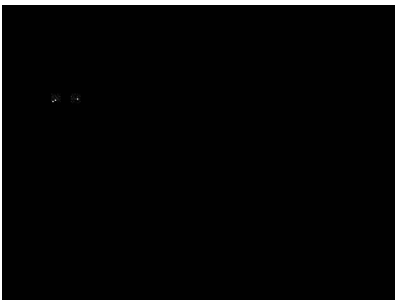

(h) Demoraum 140

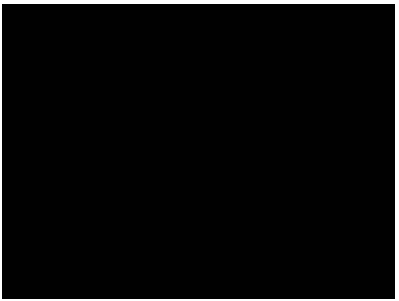

(k) Demoraum 190

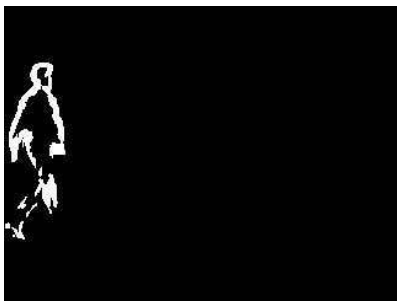

(n) Saeule 240

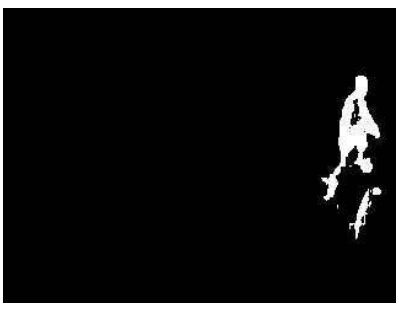

(q) Saeule 345

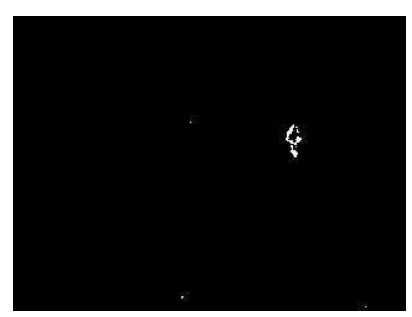

(c) Flur 200

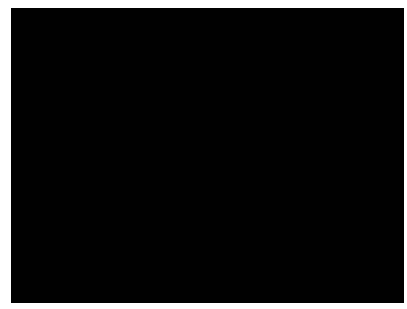

(f) Flur 500

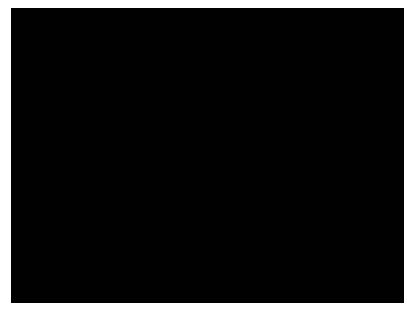

(i) Demoraum 150

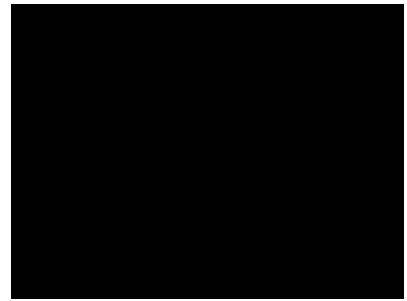

(1) Demoraum 300

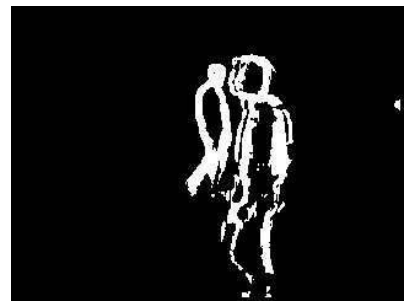

(o) Saeule 288

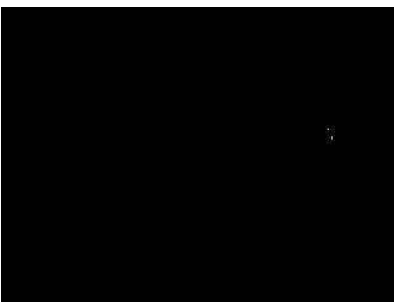

(r) Saeule 400

Fig. 1. MFG change detection masks for the Flur, Demoraum and Saeule sequences.

\section{Change Detection Results of the $M F G$}

The change detection results of the MFG are as follows:
- Flur (Fig. 1): The MFG is illumination-invariant but only some parts of the person who entered the scene are 


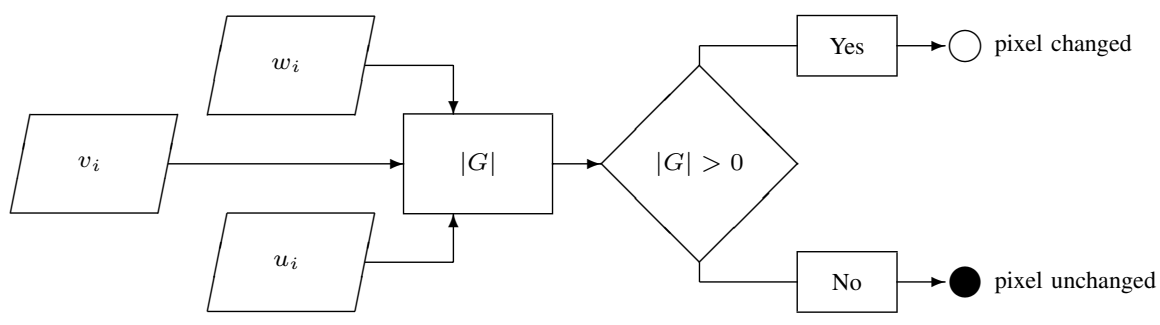

Fig. 2. The MFG method.

\begin{tabular}{|c|c|c|c|}
\hline & Flur & Demoraum & Saeule \\
\hline \hline Threshold & 0.0005 & 0.002 & 0.0005 \\
\hline Vector dimension & 9 & 9 & 25 \\
\hline
\end{tabular}

TABLE I

THRESHOLDS AND VECTOR DIMENSIONS OF THE MFG.

detected.

- Demoraum (Fig. 1): The illumination invariance is proven once again. No outliers and no contours detected as was the case for all other change detectors.

- Saeule (Fig. 1): The interiors of the persons passing the left side of the scene (see images 240 and 310) are not detected. Once they pass in front of the windows and the contrast becomes better. The slowly moving curtains are not detected. There is no influence of outliers in the change masks.

\section{Summary of Results of the $M F G$}

The MFG is illumination-invariant because of the scaling characteristics of the illumination i.e. an illumination change influences only the length of the current vector and thus the current vector and the reference vector are linearly dependent; the Gramian vanishes.

The problem of undetected moving object (persons) interiors occurs in sequences as for example in images Saeule 240. As discussed, this is due to the uniform gray-values of objects entering scenes having similar characteristics. The person interiors in Hall 190 are not detected because while one person goes to the camera, the other moves away from it. Thus the movement is parallel to the direction of vision and there is very little movement perpendicular to the direction of the vision. Thus the two consecutive current vectors $v$ and $w$ cover almost the same part of the object and thus the vectors are linearly dependent, so their Gramian vanishes.

Furthermore the change detection masks show a blurring for fast moving objects as can be seen in Saeule 288. The moving speed of both persons in the Saeule sequence may be the same, but the relative speed with respect to the static camera system is faster for the person who is closer to the camera. The contour of the fast person in the first current image and the subsequent second current image are further apart. Since the contour vectors are not scaled versions of the reference vectors and the persons interior vectors, the MFG does not vanish. Thus both contours will be visible in the change masks.

Stationary intruders are changes have not been detected as the theory predicted. Since the two consecutive current vectors $v$ and $w$ cover the same object they are linearly dependent so their Gramian vanishes.

\section{The Choice of the Color Space}

The problem of color imaging is that the color appearance of an image in general and an object in particular changes dramatically (although there is no movement in the scene) according to different viewing conditions such as media, light sources backgrounds and illumination [12]. This phenomenon causes severe problems in color control, which may influence the change detection results. Furthermore the changes are calculated pixel by pixel and not on ensembles of pixels which means that the influence of white noise will affect the results [3]. Finally, in most of the color components of different color spaces ( $Y U V, R G B, H S V$, and $\left.L_{1} L_{2} L_{3}\right)$ the object changes are not visible (see Fig. 3). Other observations are flat grey-valued components $U$ and $V$, only contours visible component $S$ and very noisy components $H, L_{1}, L_{2}$ as well as $L_{3}$.

However high-contrasted visibility of object changes in the original images is the fundament of this paper. Then by using the linear dependence change detectors the object changes can be extracted. Among the different examples of color spaces presented in Fig. 3 only the $R G B$ space provided the stated visibility of objects expectation in all of its components. Thus we will restrict ourselves to the RGB color space and the color vector model (see Eq. (1)).

Note that we assume that all color components are scaled in the same way in case illumination changes. This assumption is also best fulfilled by the $R G B$ color space.

\section{A. Color Pixel Gramian}

This section introduces the first change detector in this paper which exploits the color information in images. 


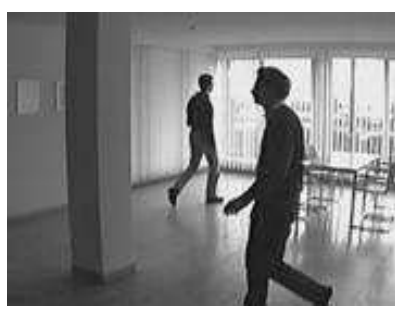

(a) $\mathrm{Y}$

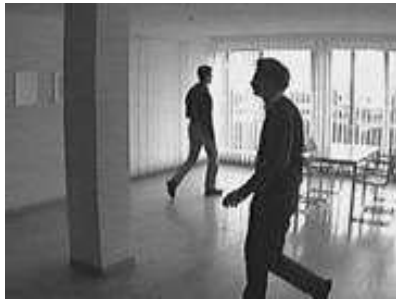

(d) $\mathrm{R}$

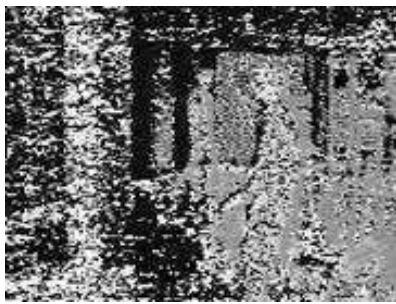

(g) $\mathrm{H}$

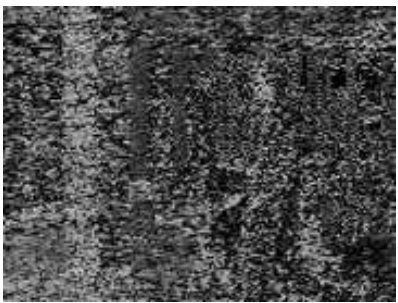

(j) L1

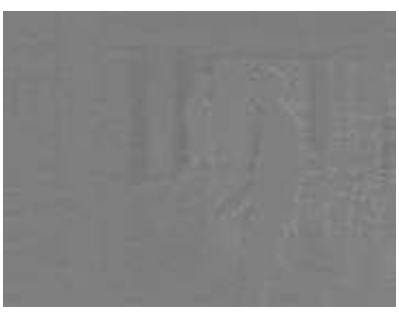

(b) U

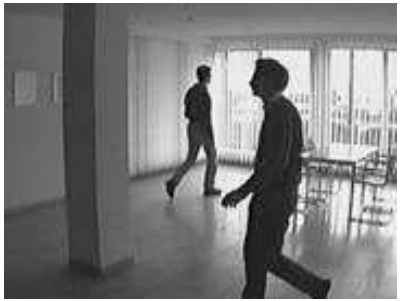

(e) G

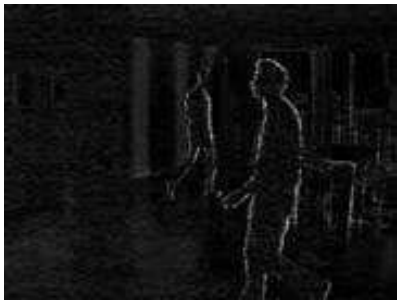

(h) $\mathrm{S}$

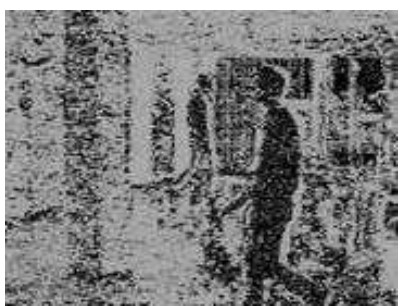

(k) L2

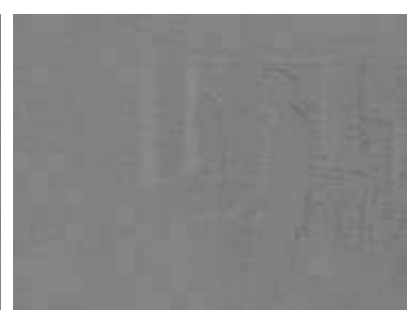

(c) $\mathrm{V}$

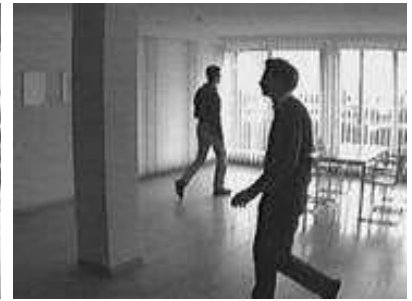

(f) $\mathrm{B}$

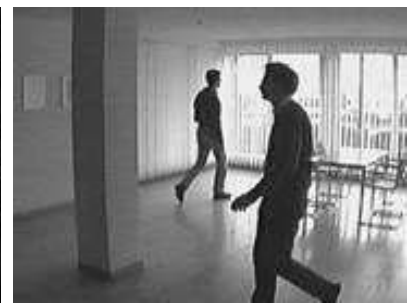

(i) $\mathrm{V}$

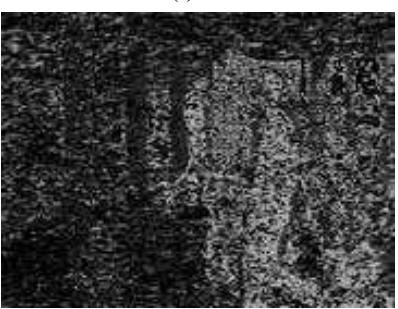

(1) L3

Fig. 3. The components of different color spaces

1) Theory of the Color Pixel Gramian: A positive point of the RGB space is that it uses a Cartesian coordinate system. The calculation of a color Gramian is therefore straightforward. The scalar product in the Gramian determinant is calculated on the color vectors (see Eq. (1)):

$$
\begin{aligned}
|G| & =\left|\begin{array}{ll}
(x, x) & (x, y) \\
x, y) & (y, y)
\end{array}\right| \\
& =1-(x, y)^{2} \\
& =1-\frac{1}{x^{2}} \frac{1}{y^{2}}(x, y)^{2} \\
& =1-\frac{1}{\left(r_{x}^{2}+g_{x}^{2}+b_{x}^{2}\right)} \frac{1}{\left(r_{y}^{2}+g_{y}^{2}+b_{y}^{2}\right)}(x, y)^{2} .
\end{aligned}
$$

This leads to the proposed Color Pixel Gramian (CPG) for unit vectors. This Gramian vanishes when the colors are the same or more generally when they are linearly dependent. When the colors are different, it does not vanish and the changing color is detected.
sectionImplementation of the CPG The Color Pixel Gramian is calculated in one accumulation summation on the reference and the current color vectors, as can be seen in Fig. 4.

The CPG requires only the threshold as a parameter since it is calculated pixel by pixel. Thus the computational cost of this method is low. When the resulting color Gramian value is greater than the threshold $T$, a change is detected and the corresponding pixel is signed as changed in the final mask, otherwise it is assumed to be 0 . The thresholds applied for the various image sequences are given in Table II.

2) Change Detection Results of the CPG: In this paragraph the results obtained by the CPG are discussed: 


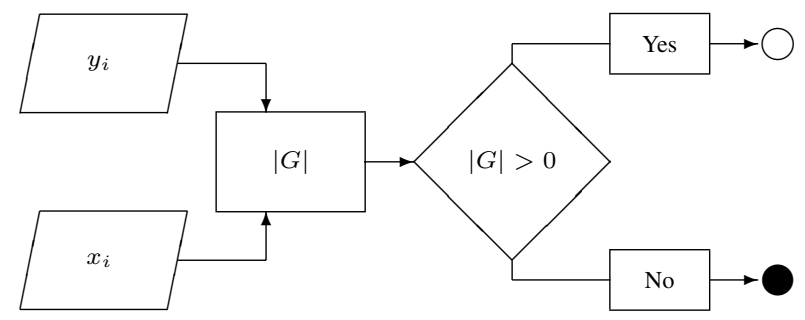

Fig. 4. The CPG method. Filled circles represent unchanged pixels, and empty circles represent changed pixels

\begin{tabular}{|c|c|c|c|}
\hline & Flur & Demo & Saeule \\
\hline \hline Threshold & 0.01 & 0.01 & 0.01 \\
\hline Vector dimension & - & - & - \\
\hline
\end{tabular}

TABLE II

THRESHOLDS OF THE CPG.

- Flur (Fig. 5): The CPG is illumination-invariant but the person who entered the scene is not detected. Noisy pixels (white noise and outliers) are visible in all change masks.

- Demo (Fig. 5): The illumination invariance is proven once again. The red seats of the chairs are detected. Noise is present in the change masks.

- Saeule (Fig. 5): Persons who passed the left side of the scene are not detected. Once they pass in front of the window the detection becomes better but their interiors have still not been detected. Almost no shadows are detected. Some moving curtains are detected. Some noisy pixels are visible in the change masks.

3) Summary of Results of the CPG: The changes are calculated pixel by pixel and thus are very sensitive to single pixel variations, as can be seen in almost all change detection masks. The CPG is illumination-invariant as can be seen in the results for the Flur and Demo sequences. Nevertheless problems occur when the observed scene reveals its color due to an illumination change, see for example the red colored seats in the Demo sequence which are all detected (see Figs. 5(i) - 5(1)) despite the fact that there is no real change present in the scene. This is due to the fact that the current red color-vector is not linearly dependent on the corresponding reference vector of the dark seat.

When the reference color-vector and the current color-vector have uniform RGB values, nothing will be detected since then the current RGB values are only the scaled version of the reference values and thus their Gramian vanishes. See for example Flur 200 there the entering person has not been detected since the colors of the reference color-vector and the current color-vector are linearly dependent.

\section{B. Vector Color Pixel Gramian}

1) Theory of the VCPG: The color pixel method CPG is calculated pixel by pixel and is thus subject to white noise. It is preferable to calculate the changes on multi-pixel regions to diminish the influence of white noise. In this paragraph the multi-pixel regions correspond to the vector model of images i.e. each color pixel is an element of these vectors. Thus, in this paragraph we propose a change detector which calculates the changes between vectors $u$ and $v$ where each component is a color pixel.

The Gramian 6 of the Color Pixel Gramian is then calculated for each element of the vectors

$|G|=\frac{1}{n} \sum_{i=1}^{n}\left(1-\frac{1}{\left(r_{x_{i}}^{2}+g_{x_{i}}^{2}+b_{x_{i}}^{2}\right)} \frac{1}{\left(r_{y_{i}}^{2}+g_{y_{i}}^{2}+b_{y_{i}}^{2}\right)}\left(x_{i}, y_{i}\right)^{2}\right)$,

where $\left(r_{x_{i}}^{2}+g_{x_{i}}^{2}+b_{x_{i}}^{2}\right)>0$ and $\left(r_{y_{i}}^{2}+g_{y_{i}}^{2}+b_{y_{i}}^{2}\right)>0$.

This is the proposed Vector Color Pixel Gramian (VCPG) for two vectors with color pixels as elements. The factor $1 / n$ insures that the same thresholds can be applied for different vector dimensions. This Gramian vanishes when the colors are the same or more generally when they are linearly dependent. In case the colors are different it does not vanish and the changing color is detected.

2) Implementation of the VCPG: The Vector Color Pixel Gramian is also calculated in one accumulation summation on the reference and the current vectors, as can be seen in Fig. 7 . The CPG requires two change detection parameters i.e. the threshold and the vector dimension. Thus the computational cost is higher than for the CPG method since the changes are calculated vector by vector instead of pixel by pixel.

When the resulting Gramian value is greater than the threshold $T$, a change is detected and the center pixel of the corresponding window is marked as changed in the final mask, otherwise it is assumed to be 0 . The thresholds and vector dimensions applied for the image sequences are given in Table III. In [4] we have proposed a method to calculate the threshold automatically. Typical thresholds were around 0.01 and good results were achieved by a vector dimension of 9 .

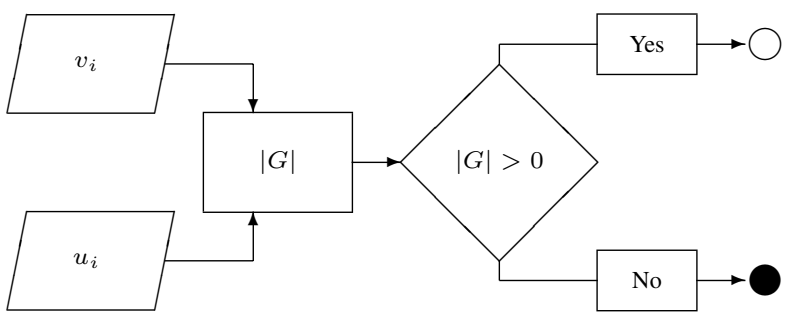

Fig. 7. The VCPG method. 


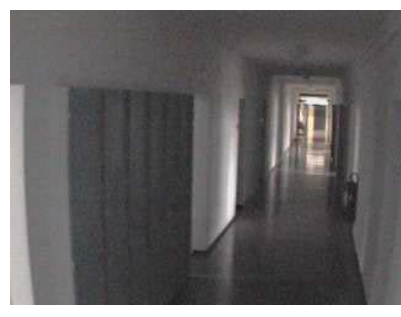

(a) Flur 0

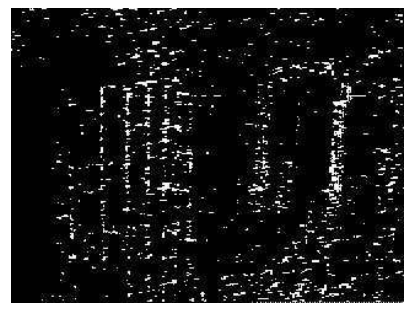

(d) Flur 221

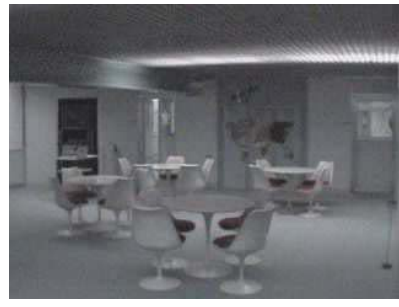

(g) Demo 0

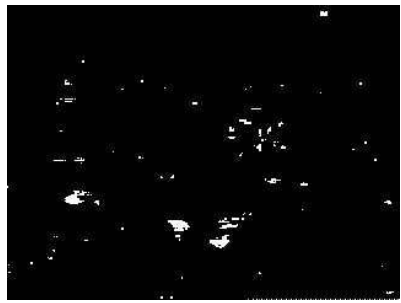

(j) Demo 160

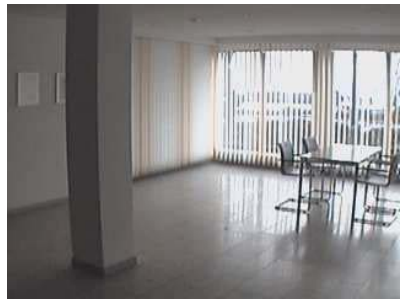

(m) Saeule 0

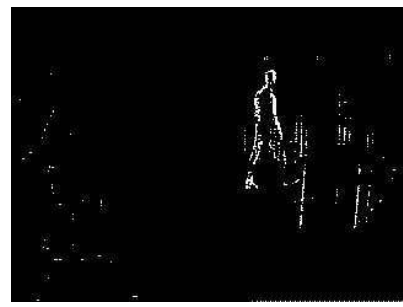

(p) Saeule 310

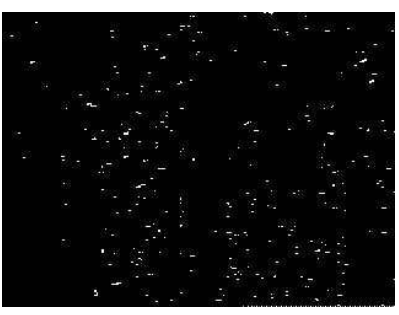

(b) Flur 185

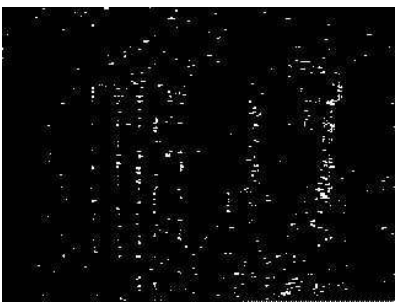

(e) Flur 260

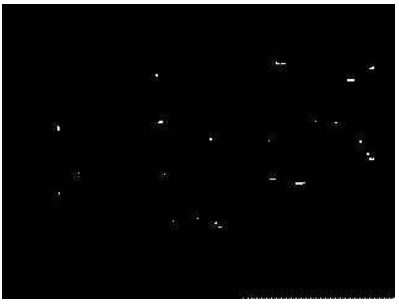

(h) Demo 140

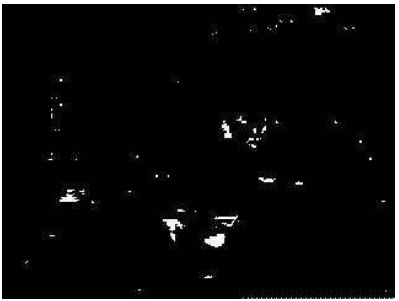

(k) Demo 190

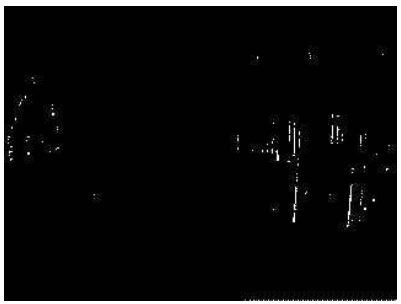

(n) Saeule 240

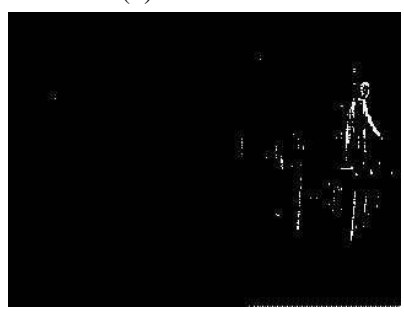

(q) Saeule 345

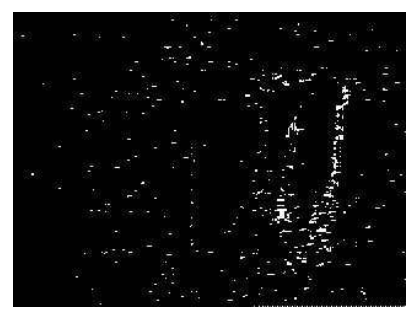

(c) Flur 200

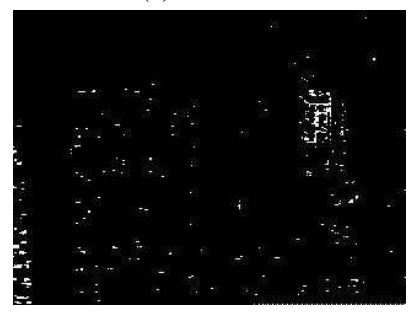

(f) Flur 500

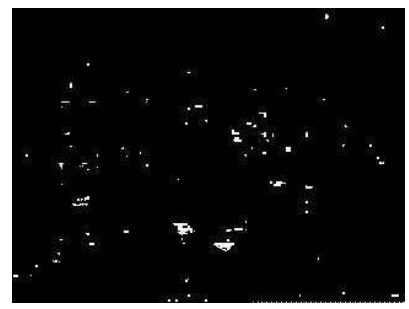

(i) Demo 150

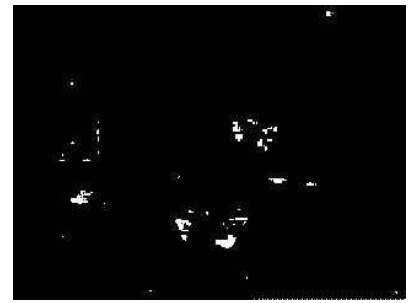

(1) Demo 300

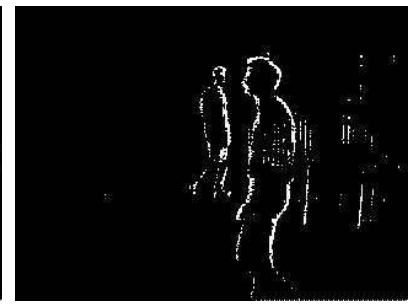

(o) Saeule 288

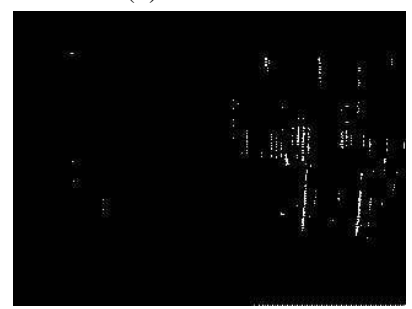

(r) Saeule 400

Fig. 5. CPG change detection masks for the Flur, Demo and Saeule sequences.

3) Change Detection Results of the VCPG: The results of the VCPG are as follows
- Flur (Fig. 6): The VCPG is illumination-invariant but the person who entered the scene is not detected. Some 


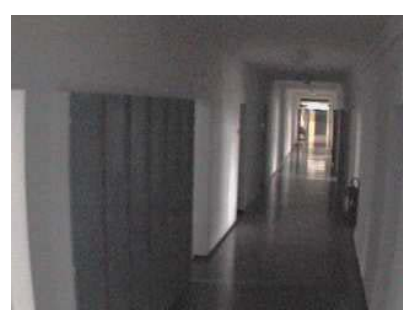

(a) Flur 0

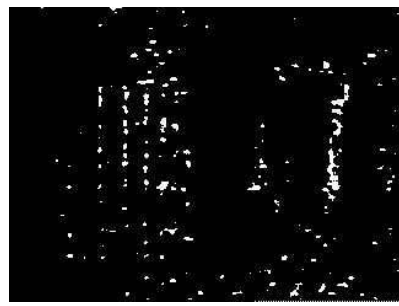

(d) Flur 221

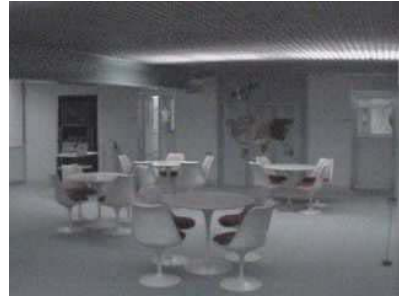

(g) Demo 0

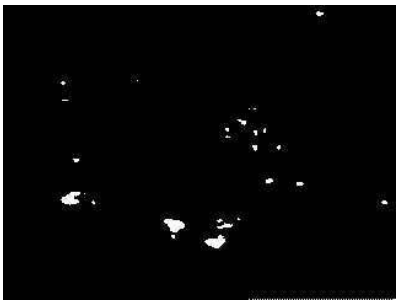

(j) Demo 160

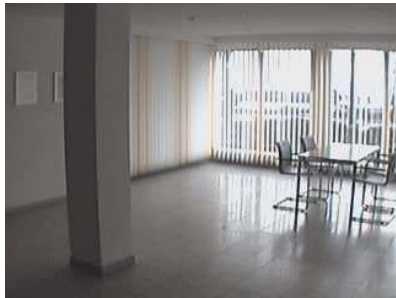

(m) Saeule 0

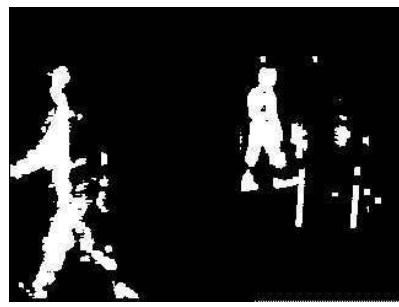

(p) Saeule 310

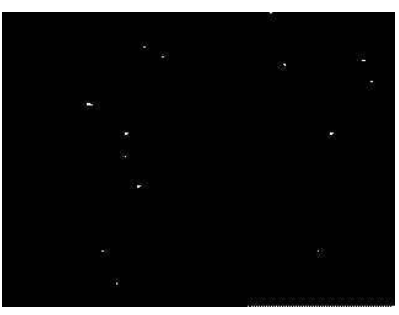

(b) Flur 185

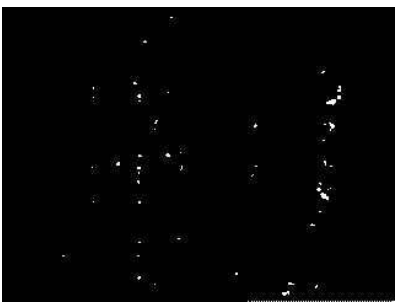

(e) Flur 260

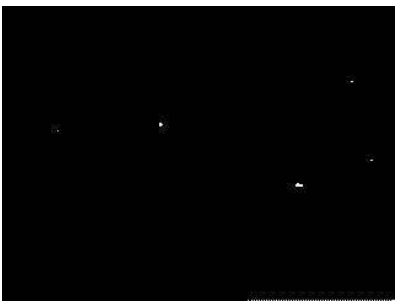

(h) Demo 140

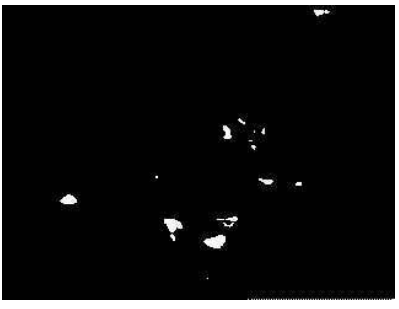

(k) Demo 190

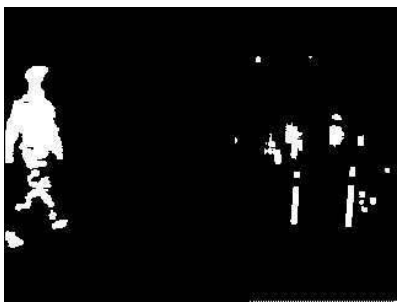

(n) Saeule 240

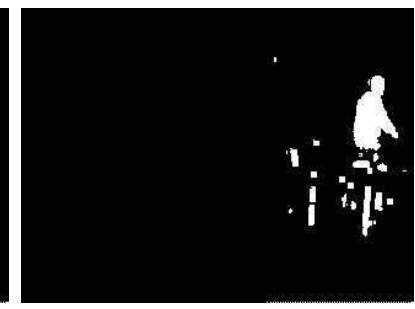

(q) Saeule 345

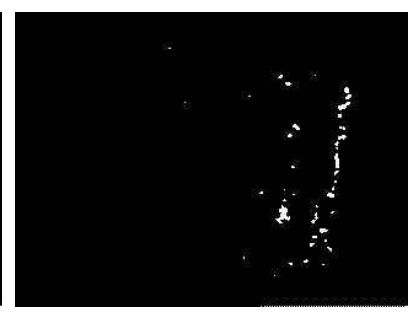

(c) Flur 200

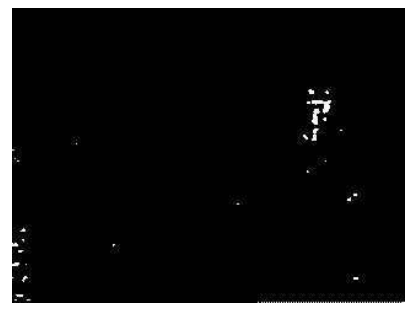

(f) Flur 500

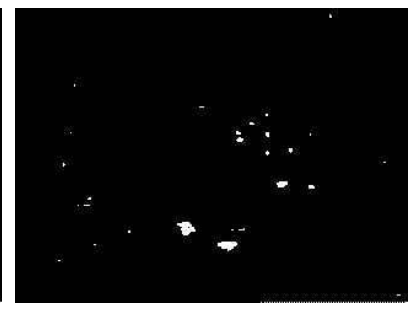

(i) Demo 150

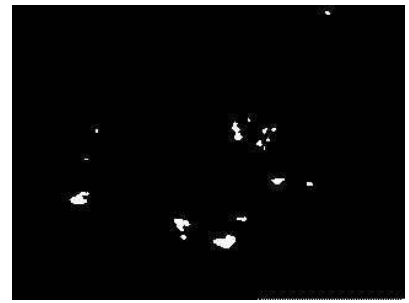

(1) Demo 300

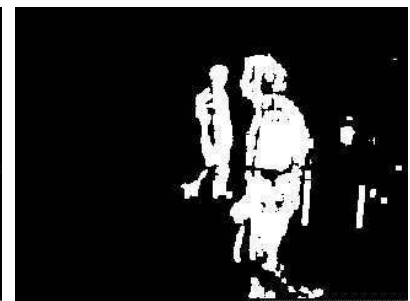

(o) Saeule 288

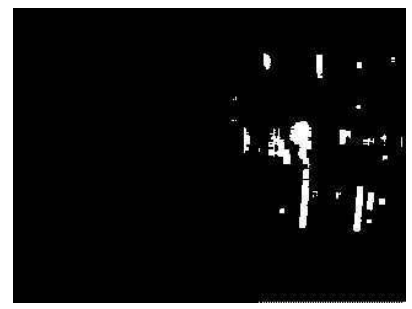

(r) Saeule 400

Fig. 6. VCPG change detection masks for the Flur, Demo and Saeule sequences.

outlier pixels are visible in the change masks.
- Demoraum (Fig. 6): The red seats of the chairs are detected. Some outliers are present in all images. 


\begin{tabular}{|c|c|c|c|}
\hline & Flur & Demoraum & Saeule \\
\hline \hline Threshold & 0.01 & 0.01 & 0.003 \\
\hline Vector dimension & 9 & 9 & 25 \\
\hline
\end{tabular}

TABLE III

THRESHOLDS OF THE VCPG. FILLED CIRCLES REPRESENT UNCHANGED PIXELS, AND EMPTY CIRCLES REPRESENT CHANGED PIXELS

- Saeule (Fig. 6): The persons are detected including their interiors. No shadows but some moving curtains are detected. Some outliers are visible in the change masks.

4) Summary of Results of the VCPG: The vector calculation improved the problem of extreme sensitiveness of the CPG method to noise. This is especially obvious in the Flur sequence (see images Flur 185 - Flur 500). However the person entering into the corridor is still not detected since the corresponding reference and current color-vectors are linearly dependent. The red seats in the Demoraum sequence are detected although this is only due to illumination change. The explanation for this phenomenon is the same as in the case of the CPG method (i.e. the current color vector is linearly independent of the reference color vector). The VCPG has also better intruder detection performance (see Saeule 240). The person interiors are detected which was not the case for the CPG method.

The behaviour of the VCPG is similar to the CPG method e.g. it also detected the red seats in the Demoraum sequence due to the same reasons.

\section{CONCLUSIONS}

We have shown that linear independence can be applied to the detection of moving objects and to the detection of color changes.

The MFG provides a very outlier-robust change detection and could be applied for moving object detection. Objects which do not move or move slowly are not, or are only partially, detected. The computational cost however is higher than for the other change detectors, since several images at the same time have to be analyzed. Furthermore the delay will increase as the number of images increases.

The CPG detects color changes. However since it is calculated pixel by pixel, single pixel variations may result in false detection. The VCPG method improved the results of the CPG method, proving that color changes can be detected between a pair of images using the Gramian determinant.

On the one hand, problems may occur for both color change detection methods when the observed scene reveals its color (or hides its color) due to an illumination change. Then, the appearing colors (or disappearing colors) will be change-detected although no real change took place. This is because the corresponding reference and current color-vectors are not linearly dependent. On the other hand, if a dark gray object enters into a light gray scene, then no change will be detected since their color vectors are linearly dependent.

Thus our research on moving object detection and color change detection will be continued but they could already open new ways in change detection. For the sake of comparison to other change detection methods on the same test images please refer to [5], [2].

\section{REFERENCES}

[1] Til Aach and Volker Metzler. Bayesian algorithms for adaptive change detection in image sequences using markov random fields. Signal Processing: Image Communication, 7(2):147-160, 1995.

[2] E. Durucan and T. Ebrahimi. Change detection and background extraction by linear algebra. The Proceedings of the IEEE, Special Issue on Advanced Video-Surveillance, 89(10):1368-381, October 2001.

[3] Emrullah Durucan. Low Computational Cost Illumination Invariant Change Detection for Video Surveillance by Linear Independence. $\mathrm{PhD}$ thesis, EPFL, Swiss Federal Institute of Technology, LTS-DE, 1015 Lausanne, 2001.

[4] Touradj Ebrahimi E. Durucan. Change detection by nonlinear grammian. In NSIP 2001, Baltimore, USA, 2001.

[5] E. Durucan et al. Change detection with automatic reference frame update and key frame detector. In NSIP 99, Antalya, Turkey, 1999.

[6] Pascal Le Quéré et al. Change detection from remotely sensed multitemporal images using morphological operators. In Geoscience and Remote Sensing, IGARSS '97, pages 252-254, August 1997.

[7] James D. Foley, Andries van Dam, Steven K. Feiner, and John F. Hughes. Computer Graphics: Principles and Practice. Addison Wesley, Reading, 1990.

[8] Y. Z. Hsu, H.-H. Nagel, and G. Rekers. New likelihood test methods for change detection in image sequences. Computer Vision, Graphics, and Image Processing, 26:73-106, 1984.

[9] Bertram Huppert. Angewandte Lineare Algebra. Walter de Gruyter, Berlin, 1990.

[10] R. Jain, D. Millitzer, and H. H. Nagel. Separating nonstationary from stationary scene components in a sequence of real world tv-images. In Proceedings of the 5th Int. Joint Conf. Artificial Intelligence,, pages 612-618, 1977.

[11] Sze-Chu Liu, Chang-Wu Fu, and Shyang Chang. Statistical change detection with moments under time-varying illumination. IEEE Transactions on Image Processing, 7:1258-1268, 9.

[12] Lindsay W. MacDonald and M. Ronnier Luo. Color Imaging. Wiley, Chichester, 99.

[13] Arun N. Netravali and Barry G. Haskell. Digital Picutes. Plenum, New York, 1995.

[14] Kurt Skifstad and Ramesh Jain. Illumination independent change detection for real world image sequences. Computer Vision, Graphics, and Image Processing, 46(3):387-399, 1989.

[15] Milan Sonka, Vaclav Hlavac, and Roger Boyle. Image Processing Analysis, and Machine Vision. ITP, Pacific Grove, California, 1998.

[16] Murat Tekalp. Digital Video Processing. Prentice-Hall, New Jersey, 95.

[17] Günter Wyszecki and Walter Styles. Color Science: Concepts and Methods, Quantitative Data and Formulae. John Wiley, New York, 1982. 